\title{
Research on the Dispersion Coefficient Measurement System based on CCD Imaging Automatic Alignment Technology
}

\author{
Cao Miao ${ }^{1}$ and Yao Qinghua ${ }^{2}$ \\ 1 Changchun University of Science and Technology, Changchun, 130022, China \\ 2 Changchun University of Technology, Changchun, 130012, China \\ 79816540@qq.com
}

\begin{abstract}
In order to measure the dispersion coefficient of glass and other transparent material rapidly and accurately, the paper studies the dispersion coefficient measurement system based on CCD imaging automatic alignment technology. On the basis of consideration of the air refractive index impacting on the measurement accuracy of dispersion coefficient, this paper rebuilds the mathematical model by the traditional dispersion coefficient measurement formula. Using the measuring angle technology of precision grating, the paper realizes the digital measurement of deflection angle of refracted ray. Sub-pixel edge detection algorithm is adopted to realize the automatic alignment of double line to single line, which has reduced artificial error of the dispersion coefficient in the measurement process. Finally, the paper combined the multichannel small collimator designed technology and the modern control technology to achieve the high precision measurement of refractive index. Demarcating the measurement system by known dispersion coefficient of optical glass, testing results and error of standard is less than \pm $2.3 \times 10^{-6}$ absolutely.
\end{abstract}

Keywords: Dispersion coefficient measurement; Air refractive index; Automatic alignment; Collimator; Image alignment

\section{Introduction}

Dispersion coefficient of the optical glass lens imaging is an important measurement of clarity, and the dispersion coefficient is calculated by the optical glass under different spectrum of refractive index. In order to ensure the quality of optical system imaging, the accurately measuring the refractive index of optical materials is more and more important. The conventional method of the optical glass prism refractive index measurement is to use an optical glass prism V refractometer. The method is the simple device, easy to measure and has a high precision test performance. However, the biggest drawback is that the human eye goes through the eyepiece and alignment. The measurement results are affected by subjective factors, and measurement efficiency is low.

In order to improve the measurement accuracy, the paper uses automatic alignment technique based on CCD images processing technology and optical shaft encoder angle measurement method. Considering air refractive index of $\mathrm{V}$ prism refraction instrument on the basis of the influence of accuracy of measurement, the new mathematical model is presented based on partial angle measuring index of refraction and dispersion coefficient. It implements the dispersion coefficient of fast automatic measurement.

\section{The New Dispersion Coefficient Measurement Model}

A ray of light passing from one medium $n$ to anther $n$ is bent or refracted from its original path according to the refraction law, which states 


$$
\frac{\sin I^{\prime}}{\sin I}=\frac{n}{n^{\prime}}
$$

For the use of a prism refractometer $\mathrm{V}$ measuring the refractive index of the glass sample schematic diagram, which is shown in Figure 1.
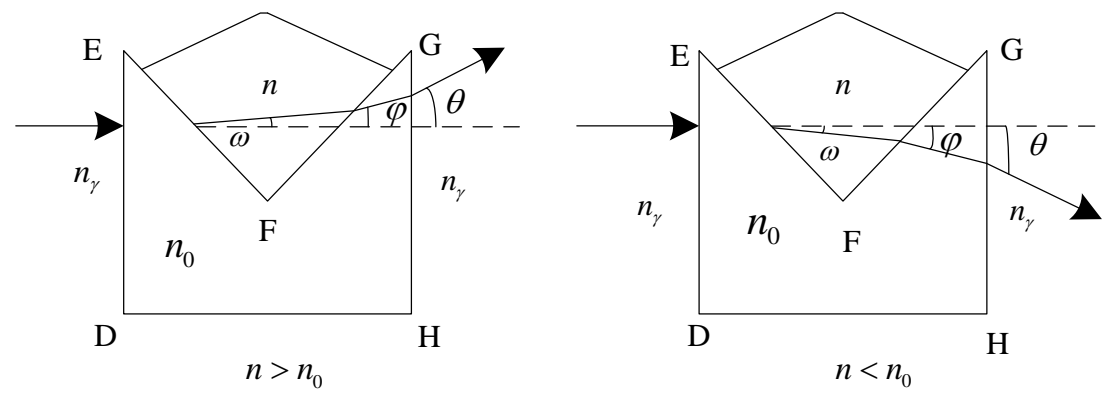

\section{Figure 1. Schematic Diagram of the V Prism Refractometer Method Measuring the Refractive Index}

The formula can be derived.

$$
\begin{gathered}
n_{0} \sin 45^{\circ}=n \sin \left(45^{\circ}-\omega\right) \\
n \sin \left(45^{\circ}+\omega\right)=n_{0} \sin \left(45^{\circ}+\varphi\right) \\
\sin \theta=n_{0} \sin \varphi
\end{gathered}
$$

By the formula (2)- (4), formula (5) can be obtained.

$$
n=\sqrt{n_{o}^{2}+\sin \theta \sqrt{n_{o}^{2}-\sin ^{2} \theta}}
$$

Formula (4) takes no account of the refractive index of air and directly derives formula (5). As shown in Figure 1, the refractive index of air is set to ${ }^{n_{\gamma}}$ and the formula (3) can be derived to equation (6).

$$
n_{r} \sin \theta=n_{0} \sin \varphi
$$

The formula (2) carries on triangular transformation.

$$
n_{0} \sin 45^{\circ}=n \cos \left(45^{\circ}+\omega\right)
$$

Separately summing the squares of formula (3) and (7) eliminating of $\omega$ is obtained.

$$
\left(\frac{n_{0} \sin 45^{\circ}}{n}\right)^{2}+\left[\frac{n_{0} \sin \left(45^{\circ}+\varphi\right)}{n}\right]^{2}=1
$$

Equation (8) is modified as follows.

$$
\sin \left(45^{\circ}+\varphi\right)=\sqrt{1-\left(\frac{\sin \theta}{n_{0}}\right)^{2}}
$$

Typing equation (6) into equation (9) can be obtained.

$$
n=\sqrt{n_{0}^{2}+n_{\gamma} \sin \theta \sqrt{n_{0}^{2}-n_{\gamma}^{2} \sin ^{2} \theta}}
$$

Formula (10) considered the effect of air refractive index on slant angle. If the air refractive index is approximating for the vacuum, namely in the formula (10) $n_{\gamma}=1$, formula (10) and formula (5) are identical.

The following is calculation of refractive index air $n_{\gamma}$. 
In order to calculate the tracing light in the atmosphere accurately, scientists conclude that air refractive index calculation formula and the accuracy can reach to $\pm 5 \times 10^{-8}$.

$$
n_{s}=\left(8091.37+\frac{2333983}{130-\sigma^{2}}+15997 \frac{15518}{38.9-\sigma^{2}}\right) \times 10^{-8}+1
$$

$\sigma$ is the wave number in vacuum, namely $\sigma=\lambda_{0}^{-1}$.

$\lambda_{o}$ is vacuum wavelength of light. Subscript s indicates the standard state, pressure $\mathrm{P}=$ $101.325 \mathrm{kPa}$, temperature $\mathrm{t}=20^{\circ} \mathrm{C}$, and the $\mathrm{CO}_{2}$ content of air is $0.03 \%$.

The refractive index of changes relationship in with temperature ${ }^{t}$ and pressure $P$ relationships is:

$$
n_{t, p}=\frac{P \cdot\left(n_{s}-1\right)}{93214.60} \times \frac{1+P \cdot(0.5953-0.009876 \cdot t) \times 10^{-8}}{1+0.0036610 \cdot t}+1
$$

Effect of changes in vapor pressure ${ }^{f}$ of the air environment to the refractive index can be expressed as:

$$
n_{t, p, f}=n_{t, p}-f \cdot\left(3.8020-0.0384 \cdot \sigma^{2}\right) \times 10^{-8}
$$

Therefore, according to measure the pressure $P$, temperature $t$, the vapor pressure $f$ in formula (12) and formula (13), the measurement environment air refractive index can be calculated.

This method calculated not accurately and inconvenience to the actual measurement. For convenience, according to the law of the People's Republic of China national metrological verification regulations on $\mathrm{V}$ prism refractometer standard conditions with environmental regulations, temperature is $t_{0}=(20 \pm 2){ }^{\circ} \mathrm{C}$, humidity is $f_{0}<85 \% \mathrm{RH}$, pressure is $P_{o}=101 \mathrm{KPa}$, and carbon dioxide content is about $0.03 \%$. Substituting the parameters $t_{0}, P_{0}, f_{0}$ into the above equation can calculate the $\mathrm{C}, \mathrm{F}, \mathrm{d}$ each spectrum refractive index of air $n_{\gamma}$ under standard environmental conditions correspond respectively 1.00027456 , $1.00027151,1.00027241$.

According to the formula (10) in F, C, d, three kinds of spectrum to measure the refractive index of the sample of the test and photometry glass sample dispersion coefficient $v$ can be obtained.

$$
v=\frac{n_{d}-1}{n_{F}-n_{C}}
$$

\section{Dispersion Coefficient Measurement System Composition and Working Principle based on CCD Imaging Automatic Alignment}

Figure 2 is a block diagram of the dispersion coefficient measurement system.

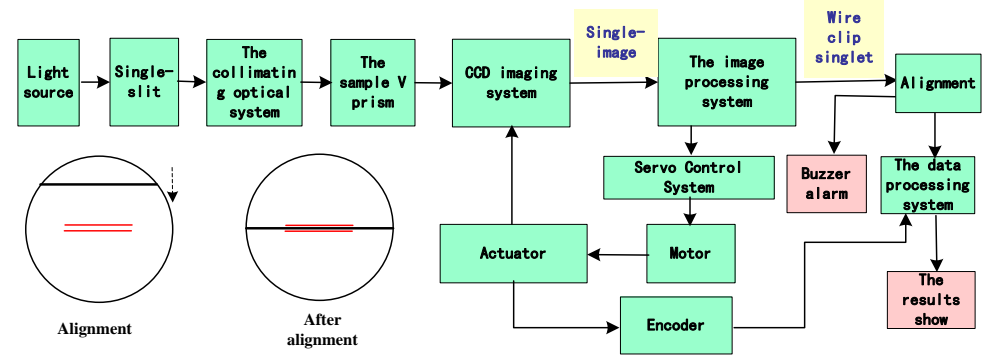

Figure 2. Dispersion Coefficient Measurement System Composition Block Diagram 
Monochromatic light source, are d light, $\mathrm{F}$ light, $\mathrm{C}$ light respectively. They are needed to give system the wavelength of light. And the wavelength is $656.3 \mathrm{~nm}, 486.1 \mathrm{~nm}, 587.6$ $\mathrm{nm}$ respectively. Working principles of the system are as follows. Monochromatic light source emits light irradiation of ground glass, illuminates singlet reticle slit, as a small collimator lens parallel to form a monochromatic light. For the small size of the mechanical structure design of the system, the $\mathrm{V}$-prism rear seat and the test sample are added to the rectangular prism, making right angle prism optical deflection $90^{\circ}$, and then through the CCD imaging lens. The slit CCD image uploaded to the computer, the operator can see the operating window on the computer screen through the slit enlarged image processing system, analysis position of the processed image and a pair of reference line automatically generated by the program. Software analysis system controls the precision servo control system via the two-wire singlet and to confirm the relative position. According to the servo system received, the drive motor rotates the control precision of the mechanical system. The image is in a middle singlet reference line, and accurate registration is achieved as shown in Figure 2. This time the system drives mechanical precision grating encoders to detect the angle measuring system, and the current angle value is calculated according to the formula (12). Finally, the calculated dispersion coefficient values are displayed.

\section{CCD Imaging and Image Process Algorithm}

This system adopts the image acquisition card for black and white image acquisition, thus the image is obtained by the acquisition card for 256 level grayscale images. Automatic machine vision alignment is realized by the CCD imaging and image process. It must be collected gray image identification, analysis and processing. The key of using $\mathrm{CCD}$ is to calculate the scribed line coordinates accurately by using image processing method.

The system adopts the subpixel edge detection algorithm to realize double single wire alignment. The basic steps are as follows.

Firstly, light is to position coarsely with the traditional sobel edge detection operator. Considering the weighted difference of digital image of each pixel of the neighboring points and gray weighted, the available sobel operator is as follows.

$$
\begin{aligned}
s(i, j) & =\left|\Delta_{x} f\right|+\left|\Delta_{y} f\right| \\
& =\left|\begin{array}{l}
(f(i-1, j-1)+2 f(i-1, j)+f(i-1, j+1))- \\
(f(i+1, j-1)+2 f(i+1, j)+f(i+1, j+1))
\end{array}\right| \\
& +\left|\begin{array}{l}
(f(i-1), j-1)+2 f(i, j-1)+f(i+1, j-1))- \\
(f(i-1, j+1)+2 f(i, j+1)+f(i+1, j+1))
\end{array}\right|
\end{aligned}
$$

The convolution operator is as follows.

$$
\Delta_{x} f=\left|\begin{array}{lll}
-1 & 0 & 1 \\
-2 & 0 & 2 \\
-1 & 0 & 1
\end{array}\right|, \quad \Delta_{y} f=\left|\begin{array}{ccc}
-1 & -2 & -1 \\
0 & 0 & 0 \\
1 & 2 & 1
\end{array}\right|
$$

Take appropriate threshold TH. Such as, if $s(i, j)>T H$, then ${ }^{(i, j)}$ is the step edge points and $\{s(i, j)\}$ is the edge image.

Secondly, as the edge is extreme value point and its second derivative value is equal to zero, this feature can be within a small neighborhood on either side of the edge points. The value gradient direction of each point is get and calculated in a small area. Its computation formula is:

$$
\theta(i, j)=\cot \left(f_{y}^{\prime} / f_{x}^{\prime}\right)
$$


Get the value $\theta$ of each point in the gradient direction in selected field, and the center pixel gradient value is compared along the gradient direction. If the gradient amplititude of neighborhood center is larger than two adjacent points along the gradient direction, then this point is the edge points and the assignment is 1 . Otherwise this point is the edge points and the assignment is 0 . The single pixel edge image is obtained. The traditional sobel operator to obtain the image edge is wide and this method can obtain image edge to single pixel level precision.

Thirdly, each edge pixel point is obtained by the above two steps of gradient value and gradient direction. The direction of the gradient can be carried out on these two values on the Gaussian curve fitting. Gauss curve expression is as follows.

$$
y=\frac{1}{\sqrt{2 \pi \sigma}} \exp \left(\frac{-(x-\mu)^{2}}{2 \sigma^{2}}\right)
$$

Logarithms is take on both sides of the formula (18).

$$
\ln y=-\frac{(x-\mu)^{2}}{2 \sigma^{2}}+\ln \frac{1}{\sqrt{2 \pi \sigma}}
$$

Formula (19) is a quadratic curve. So a binary quadratic polynomial is used to fit the edge points of neighboring domain pixel gradient of numerical curved surface fitting function. Then quadratic curve is obtained by surface fitting function to function. A quadric surface fitting function is as follows.

$$
f(x, y)=a_{0}+a_{1} x+a_{2} x^{2}+a_{3} y+a_{4} y^{2}+a_{5} x y
$$

The $\mathrm{x}, \mathrm{y}$ are for the pixel coordinates, $f(x, y)$ is the pixel gradient of $(x, y)$. The near edge of gray levels of pixels is used in the field of $3 \times 3$ and the least squares method calculates the fitting function of unknown coefficients. To solve the formula is as follows.

$$
\delta_{\min }^{2}=\sum_{x=i-1}^{x=i+1} \sum_{x=j-1}^{x=j+1}\left[f(x, y)-\left(a_{0}+a_{1} x+a_{2} x^{2}+a_{3} y+a_{4} y^{2}+a_{5} x y\right)\right]^{2}
$$

Polar coordinate is established for the center with edge points with the coefficient of quadric surface function in formula (21). Replace the curved surface function ${ }^{x},{ }^{y}$ with $\rho \cos (\theta)$ and $\rho \sin (\theta)$ respectively, and quadratic curve equation is obtained. Because of this equation is the gradient direction about the change of curvature equation. The edge of the maximum value is accurate point coordinates. Quadratic curve equation is as follows.

$$
\rho=-\frac{a_{1} \cos (\theta)+a_{3} \sin (\theta)}{2\left[a_{2} \cos ^{2}(\theta)+a_{5} \cos (\theta) \sin (\theta)+a_{4} \sin ^{2}(\theta)\right]}
$$

The coordinates of subpixel edge points can be obtained as follows.

$$
x=\rho_{g} \cos (\theta) \quad y=\rho_{g} \sin (\theta)
$$

\section{The Measurement Results}

The measurement system has been applied. Under F, C, d three spectra, low slot S FSL5 T1M1, medium slot S-T1M1 and S - BAH28 high standards of groove block of refractive index were measured, the results such as table 1 . Then the computer calculates the corresponding dispersion coefficient, the results are shown in table 2 . It can be seen from table 2, the dispersion coefficient measured value is close to the standard value extremely.

Table 1. The Measurement Data of Refractive Index

\begin{tabular}{lcccccc}
\hline $\begin{array}{l}\text { standard } \\
\text { block }\end{array}$ & $\begin{array}{c}\text { spectru } \\
\mathrm{m}\end{array}$ & $\mathrm{n}_{0}$ & $\begin{array}{c}\text { Standard piece of } \\
\text { true valuen }\end{array}$ & $\left.\theta \quad{ }^{\prime \prime}\right)$ & $\begin{array}{c}\text { Measuring the } \\
\text { average } \frac{n}{n}\end{array}$ & $\begin{array}{c}\text { absolute error } \\
E-06\end{array}$ \\
\hline Low slot & $\mathrm{d}$ & 1.510385 & 1.487566 & -9352 & 1.4875641 & -1.9
\end{tabular}




\begin{tabular}{|c|c|c|c|c|c|c|}
\hline \multirow[t]{2}{*}{ S-FSL5 } & $\mathrm{F}$ & 1.515963 & 1.492352 & -9672 & 1.4923541 & 2.1 \\
\hline & $\mathrm{C}$ & 1.507929 & 1.485421 & -9224 & 1.4854190 & -2.0 \\
\hline \multirow{3}{*}{$\begin{array}{l}\text { medium } \\
\text { slot S- } \\
\text { T1M1 }\end{array}$} & d & 1.647770 & 1.625868 & -8982 & 1.6258659 & -2.1 \\
\hline & $\mathrm{F}$ & 1.661333 & 1.638254 & -9461 & 1.6382554 & 1.4 \\
\hline & $\mathrm{C}$ & 1.642173 & 1.620734 & -8793 & 1.6207318 & -2.2 \\
\hline \multirow{3}{*}{$\begin{array}{l}\text { High slot } \\
\text { S-BAH28 }\end{array}$} & d & 1.754896 & 1.723471 & -12863 & 1.7234738 & 2.8 \\
\hline & $\mathrm{F}$ & 1.774480 & 1.736930 & -15355 & 1.7369281 & -1.9 \\
\hline & $\mathrm{C}$ & 1.746988 & 1.717863 & -11929 & 1.7178604 & -2.6 \\
\hline
\end{tabular}

Table 2. The Measurement Data of Refractive Index

\begin{tabular}{cllll}
\hline $\begin{array}{l}\text { standard } \\
\text { block }\end{array}$ & $\begin{array}{l}\text { V prism dispersion } \\
\text { coefficient }\end{array}$ & $\begin{array}{l}\text { Standard piece of } \\
\text { dispersion coefficient } \\
\text { of true value }\end{array}$ & $\begin{array}{l}\text { The dispersion } \\
\text { coefficient of average } \\
\text { measurement }\end{array}$ & absolute error $E-06$ \\
\hline $\begin{array}{c}\text { Low slot S- } \\
\text { FSL5 }\end{array}$ & 63.525403 & 70.121820 & 70.1218206 & -0.7 \\
$\begin{array}{c}\text { medium slot } \\
\text { S-T1M1 }\end{array}$ & 33.808435 & 35.715607 & 35.7156006 & -0.8 \\
$\begin{array}{c}\text { High slot } \\
\text { S-BAH28 }\end{array}$ & 27.458751 & 37.942375 & 37.9423736 & 2.3 \\
\hline
\end{tabular}

\section{Analysis of Measuring Precision}

The measuring accuracy of the dispersion coefficient depends on the refractive index measurement precision. According to formula (10), a major cause of refractive index measurement uncertainty is according to the uncertainty ${ }^{u_{1(n)}}$ of the formula about the refractive index. In addition, the refractive index measurement repeatability $u_{2(n)}$ and uncertainty are caused by other factors of measurement uncertainty ${ }_{3(n)}$, but its value is relatively small and can be ignored. Then to discuss the uncertainty ${ }^{u_{1(n)}}$ caused by the formula is to calculate the refractive index.

$$
\begin{array}{r}
u_{1(n)}=\sqrt{\left(\frac{\partial n}{\partial n_{0}}\right)^{2} u_{n_{0}}^{2}+\left(\frac{\partial n}{\partial \theta}\right) u_{\theta}^{2}} \\
n=\sqrt{n_{0}^{2}+\sin \theta \sqrt{n_{0}^{2}-\sin ^{2} \theta}} \\
\frac{\partial n}{\partial n_{0}}=\frac{n_{0}}{n}\left(1+\frac{\sin \theta}{2 \sqrt{n_{0}^{2}-\sin ^{2} \theta}}\right) \\
\frac{\partial n}{\partial \theta}=\frac{\cos \theta\left(n_{0}^{2}-2 \sin ^{2} \theta\right)}{2 n \sqrt{n_{0}^{2}-\sin ^{2} \theta}}
\end{array}
$$

The derivative of formula (24) can be obtained by formula (25).

The data in table 2 from (25) and (26) can calculated $\frac{\partial n}{\partial n_{o}}=1.00059, \frac{\partial n}{\partial \theta}=0.47576$.

Standard piece of refractive index caused by the uncertainty is $5 \times 10^{-7}$ and $u_{n_{0}}=5 \times 10^{-7} / k=5 \times 10^{-7} / 3=1.67 \times 10^{-7}$.

The error of the deflection angle theta is mainly decided by the precision of the encoder. The encoder accuracy is $\pm 1 "$. The uncertainty of measuring deflection Angle caused is $u_{\theta}=1^{\prime \prime}=4.8456 \times 10^{-6}(\mathrm{rad})$.

Take values of the parameters into the formula (24), 


$$
u_{1(n)}=\sqrt{\begin{array}{l}
\left(1.00059 \times 1.67 \times 10^{-7}\right)^{2}+ \\
\left(0.47576 \times 4.8456 \times 10^{-6}\right)^{2}
\end{array}}=2.3093 \times 10^{-6}
$$

\section{Conclusion}

In this paper, the influence of air refractive index on measuring refractive index is considered, thus affects the measured sample dispersion coefficient. A new dispersion coefficient measurement model is established. CCD imaging technology and grating displacement measuring technology are introduced in this system, and alignment type dispersion coefficient measurement system is designed. Compared with traditional measuring instrument of dispersion coefficient, the system improves the measure precision of the dispersion coefficient and the efficiency of the measurement.

\section{References}

[1] R. Yuan, Z. Zeng and L. Xiao, "Comparison of Some Methods of Measuring Refractive Index Structure Parameter [J]", Acta Optica Sinica, vol. 20, no. 6, (2000), pp. 755-761.

[2] Y.-x. Wang, Z.-w. Lu, H. Liu and H.-x. Zhang, "Application of freeform surface prism [J]", Infrared and Laser Engineering, (2007), p. 3.

[3] J. W. Liu, W. Q. Liao and H. Gui, "Exploration of the construction of online courses in engineering training education [C]", Proceeding of the 9th international conference on modern industrial training, vol. 10, (2009), pp. 202-205.

[4] X. Shang, Y. Li and X. Jiang, "Measurement of Refractive Index of Transparent Medium with Linear CCD [J]", Semiconductor Optoelectronics, vol. 33, no. 3, (2012), pp. 437-440.

[5] G. Z. Xiao, A. Adnet and Z. Zhang, "Monitoring Changes in the Refractive index of Gases by Means of a Fiber Optic Fabry-Perot Interferometer Sensor", Sensors and Actuators, (2005).

[6] Index profile of gradient refractive index ball lens using the nondestructive measurement method [A], Proceedings of Conference 7849 Optical Design and Testing [C], (2010).

[7] Metrological verification regulations of the People's Republic of China, Regulation of V prism refractometer verification China Institute of Metrology, (2005), pp. 13-23.

[8] T. Wei, Y.-K. Han, Y.-J. Li, H. L. Tsai and H. Xiao, "Temperature-insensitive miniaturized fiber inline Fabry-Perot interferometer for highly sensitive refractive index measurement", Optics Express, (2008).

[9] H.-g. Sun and J.-r. Chu, "Measurement of refractive index of plastic optical fibers", Optical Fiber Technology, (2001)

[10] P. St. J. Russel 1, "Photonic crystal fibers [J]", J. Light wave Techno 1, vol. 24, no. 12, (2006), pp. 47294749. 
International Journal of Signal Processing, Image Processing and Pattern Recognition Vol.8, No.6 (2015) 